\title{
Monolithic dielectric surfaces as new low-loss light-matter interfaces
}

\author{
Frank Brückner, ${ }^{1, *}$ Tina Clausnitzer, ${ }^{1}$ Oliver Burmeister, ${ }^{2}$ Daniel Friedrich, ${ }^{2}$ Ernst-Bernhard Kley, ${ }^{1}$ \\ Karsten Danzmann, ${ }^{2}$ Andreas Tünnermann, ${ }^{1}$ and Roman Schnabel ${ }^{2}$ \\ ${ }^{1}$ Institut für Angewandte Physik, Friedrich-Schiller-Universität Jena, Max-Wien-Platz 1, 07743 Jena, Germany \\ ${ }^{2}$ Max-Planck-Institut für Gravitationsphysik (Albert-Einstein-Institut) and Institut für Gravitationsphysik, \\ Leibniz Universität Hannover, Callinstrasse 38, 30167 Hannover, Germany \\ *Corresponding author: frank.brueckner@uni-jena.de
}

Received November 13, 2007; revised December 21, 2007; accepted December 23, 2007; posted January 7, 2008 (Doc. ID 89622); published January 30, 2008

We propose a new mirror architecture, which is solely based upon structuring of the surface of a monolithic, possibly monocrystalline, bulk material. We found that a structure of T-shaped ridges of a subwavelength grating can theoretically provide $100 \%$ reflectivity. Since no material needs to be added to the mirror device, lowest mechanical loss can also be expected. Our approach might have compelling applications as a new light-matter interface. (c) 2008 Optical Society of America

OCIS codes: $050.2770,050.6624,230.4040,230.3990$.

In many fields of physics increased research activity is currently taking place on optomechanical systems, in which a light field is coupled via radiation pressure to the dynamics of a mechanical oscillator or a free test mass [1-6]. The surface of the mechanical device provides the interface between the light field and the solid state matter. In many ongoing and future experiments, such as laser cooling of mechanical oscillators [1], optical traps for mirrors [2], generation of entangled test masses [3,4], quantum nondemolition interferometry [5], and gravitational wave detection $[5,6]$ this interface needs to provide outstanding low optical and mechanical losses. High reflectivity is demanded as lost photons result in decoherence of the light-matter quantum state. High mechanical quality factors of the test mass vibrational modes are required to reduce the influence of thermal noise [7] and to reach the quantum mechanical ground state [8]. In current approaches the surface of the mechanical device is composed of a multilayer dielectric coating. Reflectivities of up to 99.9998\% have been demonstrated [9]. To achieve high mechanical quality factors, monocrystalline materials such as quartz or silicon are used. Quality factors above $10^{9}$ were measured [10]. However, recent theoretical and experimental research revealed that multilayer dielectric coatings result in a significant reduction of quality factors [7], and that the simultaneous realization of high optical and mechanical quality is a nontrivial problem.

In this Letter we propose a highly reflective monolithic dielectric surface, which provides a solution for the great demands mentioned above. By etching T-shaped ridges into the surface of a monolithic device, perfect reflectivity of the surface can be achieved without adding any other material.

Advanced surface architectures have been suggested before to reduce the optical and mechanical loss [11]. They build on resonant waveguide gratings that comprise a periodically microstructured highindex layer attached to a low-index substrate [12-15]. Though this approach reduces the thick di- electric layer stack of conventional mirrors to a thin waveguide layer, at least one residual coating step is involved for the fabrication of such elements, thus causing a reduction of the mechanical quality. The fundamental principle of waveguide gratings is illustrated in Fig. 1(a). In case of normal incidence the three following parameter inequalities have to be fulfilled to allow for resonant reflection:

$$
\begin{aligned}
& p<\lambda \quad \text { (to permit only zeroth order in air), } \\
& \lambda / n_{H}<p \text { (first orders in high-index layer), } \\
& p<\lambda / n_{L} \quad \text { (only zeroth order in substrate), }
\end{aligned}
$$

where $p$ is the grating period, $\lambda$ is the light's vacuum wavelength, and $n_{H}$ and $n_{L}$ are the higher and lower refractive indices, respectively. In this setup, higher diffraction orders experience total internal reflection (at the boundary layer to the low-index substrate) and excite resonant waveguide modes. If $p$, the groove depth $d$, the grating duty cycle $f$ (ratio between grating ridge $b$ and period $p$ ), and the highindex layer thickness with respect to the refractive index values of the involved materials are designed properly, all transmitted light can be prompted to interfere destructively [14]. It has been shown previously that this is even possible with zero waveguide layer thickness, see Fig. 1(b) [13]. Moreover, the lowindex substrate can be reduced to a layer of some certain minimum thickness, for which evanescent transmission of the higher orders is still low, see Fig. 1(c)

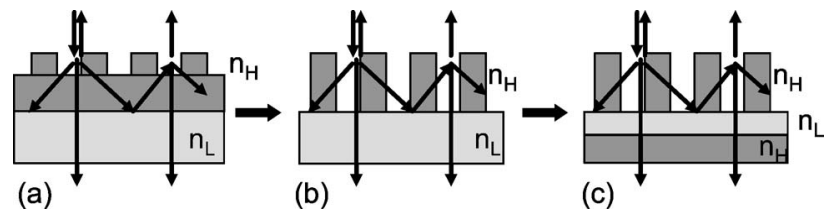

Fig. 1. Different types of resonant waveguide gratings: (a) a waveguide corrugated at its surface, (b) stand-alone highindex grating ridges, and (c) reduction of the low-index substrate to a layer. 


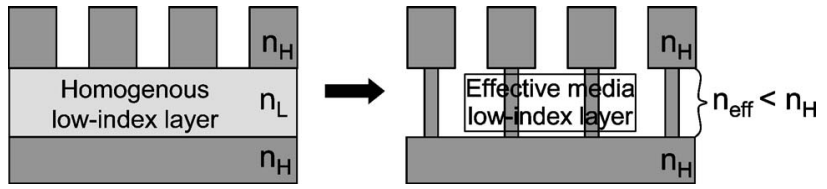

Fig. 2. Proposed architecture of a monolithic low-loss surface. The low-index layer is realized by a grating with an LDC, providing an effective medium $\left(n_{\mathrm{eff}}\right)$.

[15]. A monolithic implementation of the latter element was proposed by Ye et al. by replacing the lowindex layer with air $\left(n_{L}=1\right)$, resulting in grating ridges levitated above the substrate [16]. However, this approach appears hardly capable for applications due to its fragility and stringent production requirements.

For our new approach the low-index layer in Fig. 1 (c) is replaced by a low duty cycle (LDC) grating, which acts as an effective medium with the effective index $n_{\text {eff }}<n_{H}$. This route for a monolithic mirror setup has, to the best of our knowledge, not been proposed before. The basic idea is illustrated in Fig. 2, revealing that the LDC grating exhibits the same period as the high duty cycle (HDC) grating on top. Since the latter generates higher diffraction orders, referring to inequality (2), the description of the LDC grating as an effective medium is not obvious [17]. However, this problem is solved by a sufficiently low grating duty cycle, which makes it act very similarly to a layer of air, wherein no higher diffraction orders are allowed to propagate [inequality (3)]. The electromagnetic field inside this T-shaped periodic structure can be expressed by discrete grating modes, according to [18]. These modes correspond to the diffraction orders within a conventional resonant waveguide grating. Whereas only the fundamental mode (equivalent to the zeroth diffraction order) is propagating within the LDC grating, the HDC grating allows for higher order modes to propagate. Similar to the higher diffraction orders within a conventional waveguiding layer, the resonant excitation of these modes can result in a destructive interference of all light transmitted to the LDC grating. Thus, the monolithic T-shaped grating, as depicted in Fig. 2, can be optimized to create $100 \%$ reflectivity for particular conditions of light incident from air, which are defined by the angle of incidence, the wavelength, and the polarization state. A detailed theoretical analysis of the electromagnetic field distribution inside both gratings will be the subject of a forthcoming paper. In this approach, no material is added to the mirror device that potentially increases the mechanical loss. Very recently it was experimentally shown that a grating etched into the surface of a substrate did not reduce the substrate's quality factor, which was of the order $10^{8}$ [19].

As an example, we consider a crystalline silicon surface and calculate the parameters of a monolithic surface having nearly 100\% reflectivity for TMpolarized light (electric field oscillating perpendicular to the grating ridges) at a wavelength of $1550 \mathrm{~nm}$ for normal incidence, assuming a refractive index of $n_{H}$ $=3.5$. In case of a very LDC of the LDC grating, the effective index approaches $n_{\text {eff }} \rightarrow 1$. According to in- equalities (2) and (3), we find $443 \mathrm{~nm}<p<1550 \mathrm{~nm}$. Here, we chose a grating period of $p=700 \mathrm{~nm}$. By means of rigorous simulations [20] the four parameters of both grating regions (duty cycle $f_{\text {up }}$ and $f_{\text {low }}$, groove depth $d_{\text {up }}$ and $d_{\text {low }}$ ) can be derived. For fixed start parameters of the lower grating ( $f_{\text {low }}$ $\left.=0.25, d_{\text {low }}=2 \mu \mathrm{m}\right)$, the upper one was optimized by simultaneously varying $f_{\text {up }}$ and $d_{\text {up }}$ in the ranges from $0.4<f_{\text {up }}<0.9$ to $0<d_{\text {up }}<800 \mathrm{~nm}$, respectively. The resulting reflectivity is plotted in Fig. 3(a), revealing regions with a nearly perfect reflectivity $(99.99 \%$ up to $100 \%)$ indicated by the solid line. A highly beneficial design point is $\left(f_{\text {up }} ; d_{\text {up }}\right)$ $=(0.56 ; 350 \mathrm{~nm})$, where high reflectivity as well as convenient fabrication tolerances are found. To examine the fabrication tolerances of the lower grating for the found design point, its parameters were varied as well, ranging from $0<f_{\text {low }}<0.5$ to $0<d_{\text {low }}<2 \mu \mathrm{m}$, respectively. The plotted reflectivity in Fig. 3(b) displays a wide range of high reflectivity and supports the theoretical considerations of the preceding paragraphs. For a duty cycle smaller than $\sim 0.3$, a minimal groove depth can be found, which prevents transmission of higher grating modes to the substrate. The most beneficial parameter values arising from the simulation for TM-polarized light are, at a glance:
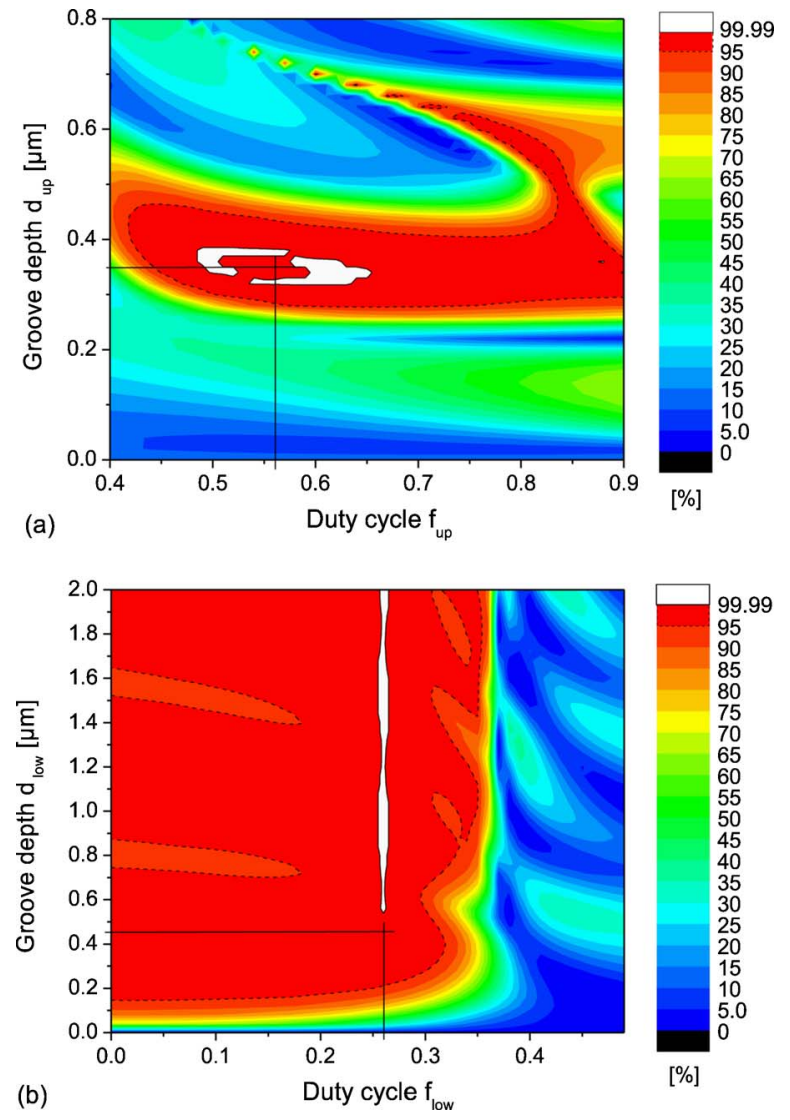

Fig. 3. (Color online) (a) Reflectivity over duty cycle $f_{\text {up }}$ and groove depth $d_{\text {up }}$ for fixed parameters $f_{\text {low }}=0.25$ and $d_{\text {low }}=2 \mu \mathrm{m}$; (b) reflectivity over duty cycle $f_{\text {low }}$ and groove depth $d_{\text {low }}$ for fixed parameters $f_{\text {up }}=0.56$ and $d_{\text {up }}=350 \mathrm{~nm}$. 


$$
\begin{array}{ll}
n_{H}=3.5 & \\
\lambda=1550 \mathrm{~nm} \\
\phi=0^{\circ} \\
p=700 \mathrm{~nm}
\end{array} \quad \Rightarrow \quad \begin{aligned}
& d_{\text {low }}=0.26 \\
& f_{\text {up }}=0.56 \\
& d_{\text {up }}=350 \mathrm{~nm} .
\end{aligned}
$$

The simulated angular and spectral properties of the device are shown in Figs. 4(a) and 4(b), revealing a $95 \%$ reflectivity for a broad wavelength range of $1550 \mathrm{~nm} \pm 175 \mathrm{~nm}$ and an angle of incidence of $\phi$ $=0^{\circ} \pm 23^{\circ}$. The reflectivity even exceeds a value of $99.99 \%$ for $1.48 \mu \mathrm{m}<\lambda<1.58 \mu \mathrm{m}$ and $\phi=0^{\circ} \pm 4.5^{\circ}$, respectively. These remarkable wide tolerances are evident due to the high index contrast between silicon and air [15].

The fabrication process is currently in progress and remains a challenging issue. One possible route is closely associated with the recently introduced fabrication of microdisks [21]. It is based on a stepwise etching process after the upper grating is defined by means of electron beam lithography. As a great benefit for the process, which can be extracted from Fig. 3 , the grating profile is not severely limited to a rectangular shape. It may vary arbitrarily within the tolerances, predicting high reflectivity.

The proposed novel monolithic mirror architecture offers new routes for many fields of experimental physics. By introducing an effective low-index layer,
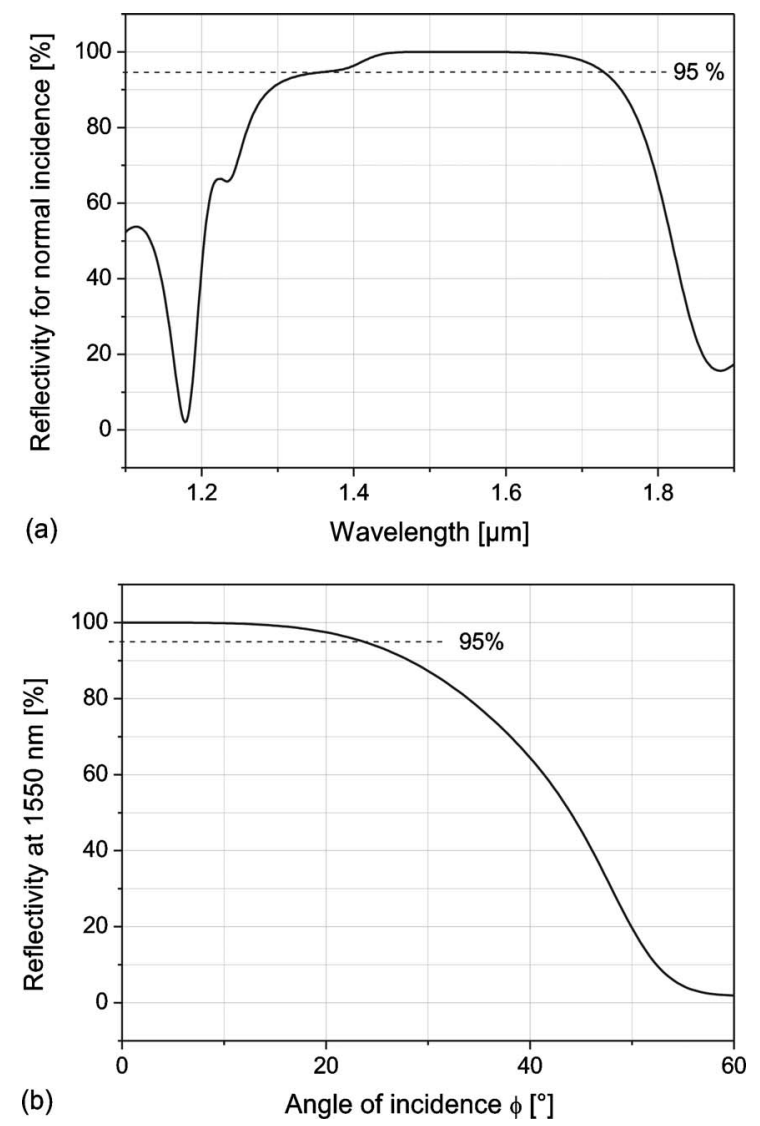

Fig. 4. (a) Spectral and (b) angular behavior of the reflectivity for the parameters given in Eqs. (4). conventional resonant waveguide devices can be advanced to prevent mechanical as well as optical loss. As a matter of course, our approach can also be expanded to other materials and wavelength regions, respectively, by means of parameter scaling. For a future paper we will address polarization effects more intensively in relation to polarizing beam splitters as well as polarization independent mirror devices.

This Letter is supported by the Deutsche Forschungsgemeinschaft within the Sonderforschungsbereich TR7.

\section{References}

1. P. F. Cohadon, A. Heidmann, and M. Pinard, Phys. Rev. Lett. 83, 3174 (1999).

2. T. Corbitt, Y. Chen, E. Innerhofer, H. Müller-Ebhardt, D. Ottaway, H. Rehbein, D. Sigg, S. Whitcomb, C. Wipf, and N. Mavalvala, Phys. Rev. Lett. 98, 150802 (2007).

3. S. Mancini, V. Giovannetti, D. Vitali, and P. Tombesi, Phys. Rev. Lett. 88, 120401 (2002).

4. H. Müller-Ebhardt, H. Rehbein, R. Schnabel, K. Danzmann, and Y. Chen, arXiv:quant-ph/0702258v3 (2007).

5. H. J. Kimble, Y. Levin, A. B. Matsko, K. S. Thorne, and S. P. Vyatchanin, Phys. Rev. D 65, 022002 (2001).

6. P. Aufmuth and K. Danzmann, New J. Phys. 7, 202 (2005)

7. Y. Levin, Phys. Rev. D 57, 659 (1998).

8. F. Marquardt, J. P. Chen, A. A. Clerk, and S. M. Girvin, Phys. Rev. Lett. 99, 093902 (2007).

9. G. Rempe, R. J. Thompson, H. J. Kimble, and R. Lalezari, Opt. Lett. 17, 363 (1992).

10. D. F. McGuigan, C. C. Lam, R. Q. Gram, A. W. Hoffman, D. H. Douglass, and H. W. Gutche, J. Low Temp. Phys. 30, 621 (1978).

11. A. Bunkowski, O. Burmeister, D. Friedrich, K. Danzmann, and R. Schnabel, Class. Quantum Grav. 23, 7297 (2006).

12. G. A. Golubenko, A. S. Svakhin, V. A. Sychugov, and A. V. Tishchenko, Sov. J. Quantum Electron. 15, 886 (1985).

13. R. Magnusson and S. S. Wang, Appl. Phys. Lett. 61, 1022 (1992).

14. A. Sharon, D. Rosenblatt, and A. A. Friesem, J. Opt. Soc. Am. A 14, 2985 (1997).

15. C. F. R. Mateus, M. C. Y. Huang, Y. Deng, A. R. Neureuther, and C. J. Chang-Hasnain, IEEE Photon. Technol. Lett. 16, 518 (2004).

16. J.-S. Ye, Y. Kanamori, F.-R. Hu, and K. Hane, J. Mod. Opt. 53, 1995 (2006).

17. P. Lalanne and D. Lemercier-Lalanne, J. Mod. Opt. 43, 2063 (1996).

18. T. Clausnitzer, T. Kämpfe, E.-B. Kley, A. Tünnermann, U. Peschel, A. V. Tishchenko, and O. Parriaux, Opt. Express 13, 10448 (2005).

19. R. Nawrodt, A. Zimmer, T. Koettig, T. Clausnitzer, A. Bunkowski, E.-B. Kley, R. Schnabel, K. Danzmann, W. Vodel, A. Tünnermann, and P. Seidel, New J. Phys. 9, 225 (2007).

20. M. G. Moharam and T. K. Gaylord, J. Opt. Soc. Am. 71, 811 (1981).

21. T. J. Kippenberg, J. Kalkman, A. Polman, and K. J. Vahala, Phys. Rev. A 74, 051802 (2006). 